\title{
Eye-movement control in visual search: How direct is visual span control?
}

\author{
ARTHUR M. JACOBS \\ Groupe Regard, Laboratoire de Psychologie Expérimentale, Paris, France
}

\begin{abstract}
In order to distinguish between the effects of low-level sensory mechanisms and those of higher level factors on eye-movement control processes, a simple letter search task was used in which cognitive load was reduced to the very minimum. The special purpose of this study was to test the visual span control hypothesis, which considers that, in such a task, eye movements are controlled as a direct function of spatial visibility limits (O'Regan, Lévy-Schoen, \& Jacobs, 1983). In a first psychophysical experiment, three methods were used to manipulate the spatial visibility limits (visual span), as measured by a psychophysical procedure: changing viewing distance, interletter spacing, and target-background similarity. The results of this experiment then were used as a reference for predicting mean saccade sizes and fixation durations in a visual search task in which the same visibility changes were made. About $80 \%$ of the variance of mean saccade sizes could be accounted for by adjustment of saccades to changes in visual span, so the visual span control hypothesis was confirmed. As to the temporal characteristics of scanning behavior, less than $50 \%$ of fixation duration variance seemed to be determined by visual span changes. Other, higher level factors, possibly related to decisional processes intervening in the triggering of saccades and the computation of their spatial parameters, might play an important role in determining fixation durations in a simple search task. The results are interpreted in relation to recent models of eye-movement control by two largely independent subsystems functioning in parallel.
\end{abstract}

The question asked in the present paper is the following: How will the spatial visibility limits that act within each fixation influence eye-movement-control processes in a visual search task in which no linguistic information is available and in which cognitive factors are reduced to a minimum?

Many investigators have tried to relate concepts such as the "perceptual span," the "useful field of view," or the "area of conspicuity" to eye-movement control in visual search and in reading (Bouma, 1978; Engel, 1977; Mackworth, 1976; McConkie \& Rayner, 1975; Paterson \& Tinker, 1947). A number of studies have also tried to decompose into perceptual, attentional, and linguistic components the factors that influence this limited area from which information is available in visual search and in reading (O'Regan, 1979; see Rayner, 1983, or McConkie, 1983, for reviews on this issue). However, except for Engel (1977), who used disks of variable sizes as stimuli and who was not directly interested in saccade size and fixation duration, there has been only one systematic attempt to predict eye-movement parameters directly on the basis of quantitative measures of the visuosensory constraints imposed on eye-movement behavior.

This research was supported by a grant from the "Studienstiftung des Deutschen Volkes." I would like to thank the members of the Groupe Regard, especially Kevin O'Regan and Ariane Lévy-Schoen, for their helpful discussions. The author's mailing address is: Groupe Regard, Laboratoire de Psychologie Expérimentale, EPHE, 3e section, EHESS, associé au CNRS, 28 rue Serpente, 75006 Paris, France.
In that attempt, which was designed to distinguish between the effects of early visual mechanisms and higher level processes on perceptual span, O'Regan, LévySchoen, \& Jacobs (1983) first proposed a definition of visual span as the size of the region around the eye's fixation point in which letters can be recognized with a given accuracy, without the use of any contextual information. The limits of this span depend on purely visual factors, such as viewing distance, letter similarity, or interletter spacing, and can be estimated using the model proposed by O'Regan (1983). Under the simple hypothesis that there must be a relationship between the limits of this span and eye movements in reading or visual search, saccade sizes should be found to parallel changes in visual span, induced by varying visibility conditions (viewing distance, letter spacing). However, O'Regan, et al. (1983), using a reading task, and Lévy-Schoen, O'Regan, Jacobs, and Coeffe (1984), using a complex visual search task, failed to show a direct dependence of eye-movement parameters on visual span changes. It was suggested that, in these tasks, higher level factors related to linguistic processing and cognitive load, determined eye-movement behavior more than did the spatial sensory limits constraining visibility of letters at each fixation.

The idea behind the present research, which continues these studies, was the following: If cognitive load could be reduced to the very minimum in a visual search task, then the control of saccades should depend directly on the sensory constraints limiting the number of letters visible around the eye's fixation point. The global hypothesis here is that, under such conditions, each saccade should bring 
the eye to a zone where new visual information can be gathered, that is, the limit of the visual span. As a consequence, if visual span is changed as a function of the experimental visibility condition, then saccade sizes should, on average, change in parallel. This extreme hypothesis, which considers eye-movement behavior as a direct function of spatial sensory limits, will be called the "visual span control hypothesis." The purpose here is to evaluate to what extent the experimental data disagree with such a deterministic prediction.

\section{VISIBILITY EXPERIMENT}

\section{Method}

In the present letter-detection task, three experimental methods were used to vary visibility conditions. These methods, which had already been shown to create significant changes of visual span in other studies, were: changing viewing distance, changing interletter spacing, and changing the graphic similarity between the target and the background (Jacobs, 1985a; O'Regan et al., 1983).

Two levels of viewing distance, 60 and $120 \mathrm{~cm}$, and two levels of interletter spacing were used. In the normal spacing condition, the letters were separated by a space of 4 pixels; in the double spacing condition, the blank separating two adjacent letters was 8 pixels in size.

Target letters were presented within a line of 79 xs ( 50 for doublespacing condition) on a computer screen. There was one single type of target letter used for a block of trials, and the subject was informed what the target was. Thus, effects on performance created by confusing target letters were obviated. The target letter was presented at varying eccentricities from the fixation point, and the subject's task was to report if the target appeared to the right or to the left of the fixation point or if there was no target within the line of $\mathrm{xs}$.

Four levels of target-background similarity were chosen using the method of letter-similarity estimation proposed by Keren and Baggen (1981). Figure 1 gives the target stimuli and the background (with the corresponding letter matrices) used (the lines are shorter than the original 79 letters). Note that the two targets most similar to the background, denoted " $k$ " and " $x$ '," were created by changing the letter matrices used by the computer: for example, the most similar target, $x^{\prime}$, was obtained by changing just one pixel of the normal matrix for the letter $x$.

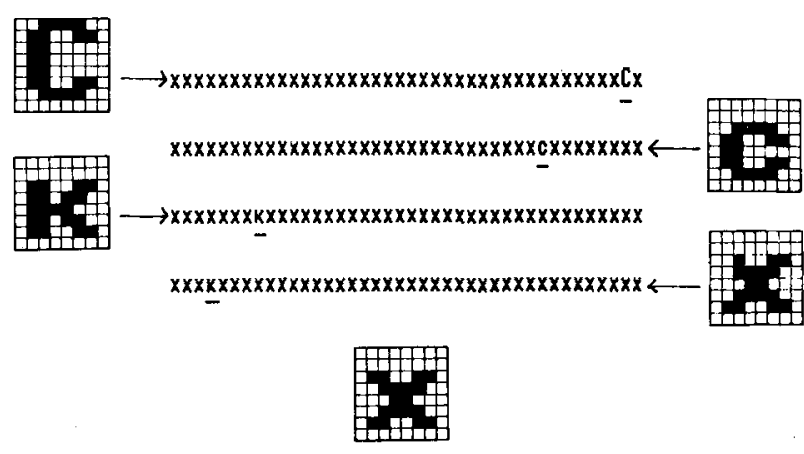

Figure 1. Examples of stimulus material and of letter matrices used in the visibility and visual search experiments. Each of these lines contains one of the four target letters representing the four levels of increasing graphic similarity (from top to bottom) between target $(C, c, k$ and ' $x$ '") and background $(x)$ which have been chosen using the estimation method proposed by Keren and Baggen (1981). Note the differences between the modified letter matrices of the two most similar targets ( $k$ and " $x$ " ") and those for the background $x$.
A series of pilot experiments were undertaken to find a set of target letters that had the greatest possible range of target-background similarities. The " $k$ " (with ascender cut) and " $x$ "' were invented because these pilot experiments had shown that, given the background of xs and our special method to measure visual span (see below), most targets (without ascenders or descenders), such as a, $c, e, m, n, o, r, s, u, v$, and $w$, did not differ substantially in peripheral detectability.

Adaptive psychophysical procedure for measuring visual span. A computer-controlled adaptive psychophysical method, functioning in real time, was used to choose the eccentricities at which the target letter was to be presented. The procedure used was similar to the one known as "APE"' (Watt \& Andrews, 1981) and converges faster than the "PEST" procedure (Taylor \& Creelman, 1967) (for details, see Humbert, 1985). The principle was the following: At the beginning of each experimental block, the target stimulus was presented 10 times at the central position, indicated by the fixation cross, in order to familiarize the subject with the target and to ensure that the target was always recognizable in central vision. The computer then presented the target once at each of the extreme eccentricities $(-38,+38,-1,+1$ character positions from the central fixation point, which was at position 40 in a typographic mode using 80 columns or character positions per line). Note that the eccentricities -39 and +39 were ruled out because we wanted to ensure lateral masking to be the same across eccentricities. This required that target letters always be flanked on both sides by at least one $x$. On the other hand, given that the target $\mathrm{C}$ proved to have a bigger visual span (in preexperiments) than the maximum eccentricity possible on the display screen, a particular technique was used in this case: the fixation point was shifted to the left (position 0 on the screen), and the target was always presented to the right of the fixation point. Thus, the maximum eccentricity possible doubled to 78 characters.

On the basis of the responses given by the subject for each of the four extreme presentation eccentricities, the computer calculated a first estimation of the spatial threshold (eccentricity) corresponding to a response probability of $66.7 \%$, given the chance level of $1 / 3$. It then presented the target letter at the eccentricity resulting from this computation. The program used the "probit" technique (Finney, 1947; Watt \& Andrews, 1981) for fitting the data to a theoretical psychophysical curve. After the subject's next response, the algorithm again computed the most probable threshold eccentricity, given all the data points sampled up to this moment, and fitted by the probit method. It then presented the target at the corresponding eccentricity, and so on. This procedure was repeated 50 times. The results in the literature, as well as in computer simulations done by Humbert (1985), show that the precision of this adaptive procedure reaches a stable plateau by 50 trials. In total, 50 trials plus 25 randomly mixed-in control trials (without any target) and 30 training trials were used for one experimental block.

There were four subjects, all members of our research group who were well-practiced in visibility experiments. A repeated measures experimental design was used, in which each subject served in all of the 16 visibility conditions ( 2 distances $\times 2$ spacings $\times 4$ levels of target-background similarity) but in different orders. To obviate effects due to associations between the viewing distance, spacing, and target-background similarity variables, four Latin squares were used, as well as a counterbalancing technique similar to the one described by O'Regan et al. (1983).

The experiment was controlled by a BBC and an IMS microcomputer. Stimuli appeared on a VELEC VS display terminal that used a P4 phosphor. The character matrices of the BBC computer were of $8 \times 8$ pixels; their width subtended $1 / 3^{\circ}$ of visual angle at a viewing distance of $60 \mathrm{~cm}$ and $1 / 6^{\circ}$ at a viewing distance of $120 \mathrm{~cm}$. Lighting conditions were photopic and were kept identical across subjects, as were screen brightness and contrast. The display background luminance was about $67 \mathrm{~cd} / \mathrm{m}^{2}$; stimulus luminance was about $125 \mathrm{~cd} / \mathrm{m}^{2}$ (high contrast).

The subject's eye movements were monitored in order to verify that his or her eye did not move from the fixation mark during the 
trial. A photoelectrical scleral reflection technique was used (O'Regan et al., 1983). If a fixation error ( \pm 1 letter position from the fixation mark) was detected, the trial was automatically rejected by the computer and replaced, at random, by another one.

After the eye-movement calibration phase at the beginning of each block, the subject fixated a small gap between two fixation lines and pressed a button to begin the trial. The stimulus line was then flashed on for $150 \mathrm{msec}$ and the subject gave his or her response using one of three buttons (target left, target right, or no target). Although the short exposure duration of $150 \mathrm{msec}$ did not need to be employed for the purpose of preventing eye movements, since they were verified, this duration was chosen for reasons of compatibility with previous results (O'Regan et al., 1983).

\section{Results and Discussion}

For the reasons discussed by O'Regan et al. (1983) (cf. Morrison \& Rayner, 1981), the values of visual span given in Figure $2(a+b)$ are measured in number of letters identifiable on each side of the fixation point, and not in
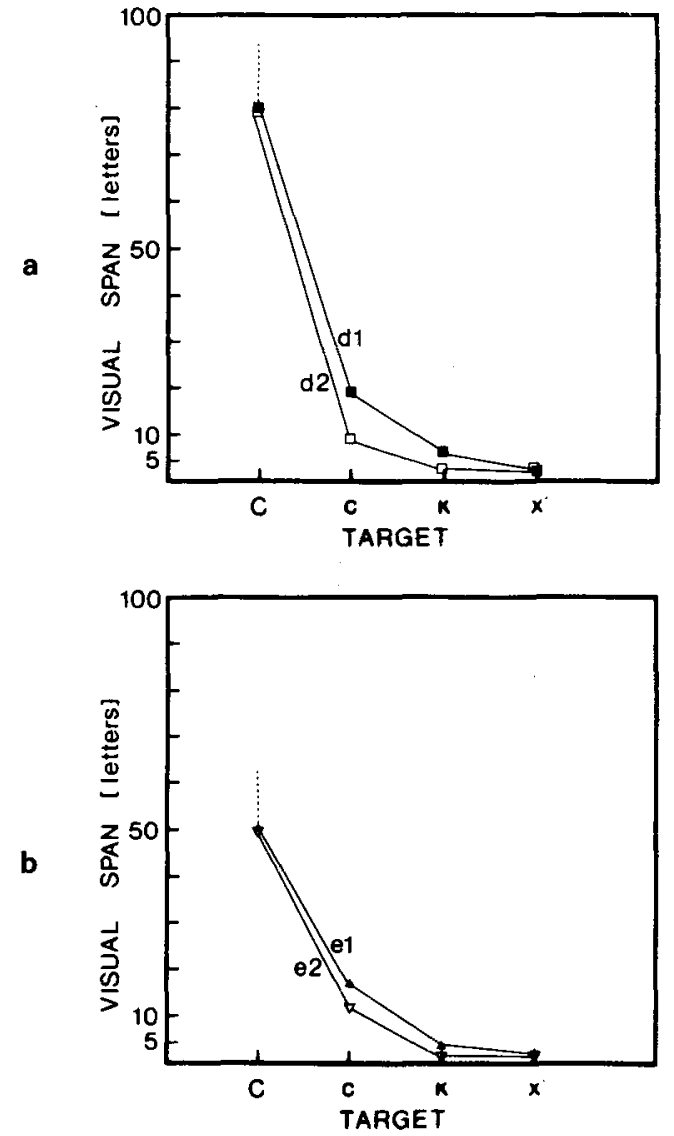

Figure 2. Visual span in the visibility experiment as a function of target-background similarity and viewing distance (a) and as a function of target similarity and interletter spacing (b). d1 and d2 are the two viewing distances $(60$ and $120 \mathrm{~cm}), \mathrm{e} 1$ and $\mathrm{e} 2$ are the two spacings ( 4 and 8 pixels), and $C, c, k$, and " $x$ '" are the four target letters representing the four levels of increasing targetbackground similarity. Span is measured as the number of letters visible on each side of the fixation point with a response probability of better than $50 \%$. It is obtained by an adaptive psychophysical procedure, similar to the APE method proposed by Watt and Andrews (1981), developed in our research group by Humbert (1985). degrees. They are obtained directly by the adaptive psychophysical procedure described above. In particular, the values plotted in this figure correspond to the last threshold estimation given by the adaptive algorithm, which is the threshold corresponding to the 50th trial. Note that, as in O'Regan et al. (1983), the "span" really is a half-span, since it refers to the eccentricity on either the left or the right side of the fixation point at which the target letter can be identified with $50 \%$ correct responses.

Figure 2a shows visual span as a function of targetbackground similarity (target letter) and of viewing distance; Figure $2 b$ shows visual span as a function of similarity and spacing. Measured in number of letters, span decreases systematically as target-background similarity increases $[F(3,9)=546.61, p<.0001]$ and as viewing distance $[F(1,3)=16.1, p<.05]$ or letter spacing $[F(1,3)=21.3, p<.05]$ increases. These results replicate the findings of O'Regan et al.'s (1983) visibility study, which showed that visual span declines as visibility conditions become more difficult. There is no significant interaction between the three factors, but a ceiling and a floor effect are obtained for the two extreme targets: the letter $\mathrm{C}$ was, independently of the visibility condition, always visible, within the line of $x s$, beyond the maximum eccentricity possible with our computer screen (79 positions for normal spacing and 49 for double spacing). This is indicated by the dashed lines. In contrast, the visual span of the letter " $x$ "' was, in all the experimental conditions, never better than one or two character positions from the fixation point. This is why no distance or spacing effect could be found for these two targets. However, for the two other targets, distance and spacing effects are clear, as shown by a restricted statistical analysis: for the letter $c$, an $F(1,3)$ of 336.6 , $\mathrm{p}<.0001$, is obtained for viewing distance and an $F(1,3)=31.3, p<.025$, is obtained for spacing. For the $\mathrm{k}$, an $\mathrm{F}(1,3)=21.6 ; \mathrm{p}<.05$, is obtained for distance and an $F(1,3)=16.8$, p. $<.05$, is obtained for spacing.

The case of the two extreme letters is, nevertheless, of special interest for the visual search study to be described below. Given the perfect peripheral detectability of the $\mathrm{C}$, it was of interest to determine what the eyes would do in a scanning situation in which the presence/absence of the target was visible from all positions in the line. The case of the " $x$ " " was exactly the opposite: would the eyes make saccades of the size of one or two letters in this condition, a very unnatural scanning mode?

Of course, the important point here is that each of the three factors has shown a systematic effect on visual span, so the results can be used as a reference for the visual search experiment that follows.

\section{VISUAL SEARCH EXPERIMENT}

The visibility experiment showed that visual span declines strongly when target-background similarity is increased and is reduced also when viewing distance and 
letter spacing are doubled. Under the extreme hypothesis of direct oculomotor control by visual span, saccade sizes and fixation durations are expected to adjust to the observed changes in span when visibility conditions vary. The present experiment, using exactly the same subjects, stimuli, and visibility conditions as in the previous experiment, tests this hypothesis.

\section{Method}

As in the visibility experiment, a homogeneous background consisting of lines of xs, within which a known target letter was hidden at randomly varying positions, was used. Thus, basically, cognitive load was reduced to a two-element-discrimination task. As an approximation, one can consider that, at each fixation during the scanning of these lines, the cognitive effort was reduced to the decision of determining whether a target letter was present or absent within the zone of visibility (Jacobs, 1985b).

The two viewing distances, interletter spacings, and four target letters used in this experiment were the same as those used in the visibility study. The subjects scanned 15 lines consisting of xs and targets. Each set of lines had the following structure: Five lines, whose position in the set was permuted, contained either one or two targets. The positions of the targets in the line were randomized, with the constraint that there be only one target in each half of the line. The first line of a set always contained one or two targets to serve as reminders to the subject. The other 10 lines contained only xs. It is important to note that only one type of target letter was presented within the same set of lines, and that the subject was informed of what the target was. Thus, any effects due to confusion between targets were prevented: we were interested in the sensory mechanisms of peripheral vision, and not in the effects of positive or negative set size.

The order of presentation of the two distances, spacings, and four levels of target-background similarity followed the same experimental design as in the visibility study.

After an initial calibration phase, the subject, sitting in an adjustable chair with his or her head stabilized by a chin-/foreheadrest, scanned the set that appeared, line by line, on the computer screen. The display technique used was the same as that used by $O$ 'Regan et al. (1983), in which the subject's return eye movement triggers the disappearance of the line just scanned and the appearance of the next line. This technique provides precise measures of horizontal eye movements and subjects adapt to it easily. As in the visibility experiment, eye movements were recorded using a photoelectric scleral reflection method, and eye position was sampled by the computer every $10 \mathrm{msec}$. While the subject was scanning the lines, the computer continuously corrected the initial calibration for slow shifts caused by head movements or other artifacts. This correction was done by assuming that each return sweep started from near the end of each line and brought the eye to a fixed position at the beginning of the next line. The automatic recalibration disturbed the recordings near line beginnings and line endings, so data for such points were not included in the analysis. Relative accuracy of estimation of the size of the saccades made was guaranteed to be the same across subjects and conditions and of the order of one character space.

To count the number of targets that occurred, and not miss any target, the subjects were instructed to scan the lines, from left to right, just as they would in reading. In pilot studies, the difficulty of the task of remembering exactly the number of targets seemed to influence eye movement parameters. Since such a cognitive effect upon eye movements was not wanted, the subjects were instructed and trained to press a button each time a target was encountered, thus freeing them of the burden of remembering. This task was found to have no effect on eye movements, and the subjects adapted very easily to it. In any case, the eye-movement data presented here were selected only for lines in which no target occurred. After each experimental block, the correctness of the subjects' performance in terms of number of targets detected was assessed. To discourage unattentive scanning behavior, the subjects were informed that if there were omissions the experimental run would have to be repeated. Thus the instructions stressed accuracy of performance, rather than speed.

Saccade sizes and fixation durations were analyzed by the computer according to the following principle: A saccade was defined as a change in eye position of more than one character space, taking less than $50 \mathrm{msec}$ and giving rise to a fixation lasting more than $50 \mathrm{msec}$

The four subjects, all well-practiced, were the ones used in the previous experiment on visual span. The lighting and other experimental conditions were also the same as those of the previous experiment.

\section{Results and Discussion}

Analysis of the spatial adjustments of search behavior (saccade sizes). Of interest here was ocular behavior while the lines were being scanned in search of an expected target, and not the adjustments of the eyes when a target fell within the peripheral field of view. Thus, only the lines containing no targets were selected for analysis.

The mean lengths of the forward saccades (progressions) were computed for each subject and each experimental condition, as were mean fixation durations. Note that only fixations following forward saccades were selected for this analysis, since only predictions about progressions were made. As in the preceding experiment, a three-factor repeated measures ANOVA was computed. This reveals that neither distance $[\mathrm{F}(1,3)=.338$, n.s. $]$ nor spacing $[F(1,3)=5.8$, n.s.] had a significant effect on mean progression sizes, although there might be a slight tendency toward smaller saccades when spacing was doubled. However, when target-background similarity increases, there is a strong effect on saccade sizes $[F(3,9)=110.5, p<.005]$ : the more similar the target is to the background, the greater the decrease in progression sizes. Recent studies by Prinz and Kehrer (1982) and Rayner and Fisher (1985) showed results consistent with these findings: search performance declined significantly when target-background similarity was increased. However, array density (item spacing) in Rayner and Fisher's study did not affect performance as much as did target-background similarity, and in Prinz and Kehrer's study it had no effect at all.

The fact that neither distance nor spacing showed significant effects on mean progression amplitudes is not consistent with our working hypothesis of direct visual span control, given that both factors influenced directly visual span as measured in the preceding visibility experiment. However, the effects of distance and spacing in the visibility experiment were weak, relative to the similarity effect. Probably, the strong variability of the eye-movement data (to be discussed later), in contrast to the "zero variability" of the psychophysical threshold measures (one single threshold or visual span value is measured by the adaptive procedure for each experimental condition, as pointed 
out above), was responsible for the lack of an effect of these two factors on saccade sizes.

To test the hypothesis of direct control of saccade sizes by visual span in a more straightforward manner, the measure of visual span for each subject and each experimental condition was compared to the corresponding value of mean progression amplitude. This is done in Figure 3.

Under the extreme working hypothesis, one would predict that the data points would fit the theoretical diagonal line perfectly. This turns out to be not completely true. Nevertheless, a direct linear relationship between visual span and mean progression size is evident. Each of the four correlation coefficients (Bravais-Pearson) is superior to .9 , and they are all significant at the $1 \%$ level. The coefficient of determination, $\mathrm{r}^{2}$, which is an indication of the amount of variance of one variable accounted for by the variance of another, is, in every case, superior to .8. So one can consider that about $80 \%$ of the variance of progression sizes are determined by changes in visual span.
Eighty percent of variance in mean progression sizes explained represents, in any case, a good result, and thus the hypothesis of direct oculomotor control by visual span seems to be confirmed for this kind of task. However, one point attracts attention in considering the correlation diagrams: Apparently, the larger the visual span, the less progression sizes follow the theoretical line. To verify this, and to obtain a more precise idea of how progression sizes adjusted locally to the changing visibility conditions, relative frequency histograms were plotted for saccade sizes (measured in number of letters) as a function of viewing distance (Figure 4a), interletter spacing (Figure $4 \mathrm{~b}$ ), and target-background similarity (Figures $4 c-4 f)$.

The interesting finding here, which confirms the previous suggestion concerning the correlation diagrams, comes from the c-f plots in Figure 4. One can easily see that the variance of progression sizes decreases rapidly as targets become more similar and, of course, as span declines (the variance/mean ratios are: $79 \%$ for the $\mathrm{C}$, $72 \%$ for the $c, 56 \%$ for the $k$, and $48 \%$ for the " $x$ "' $\operatorname{tar}$ -

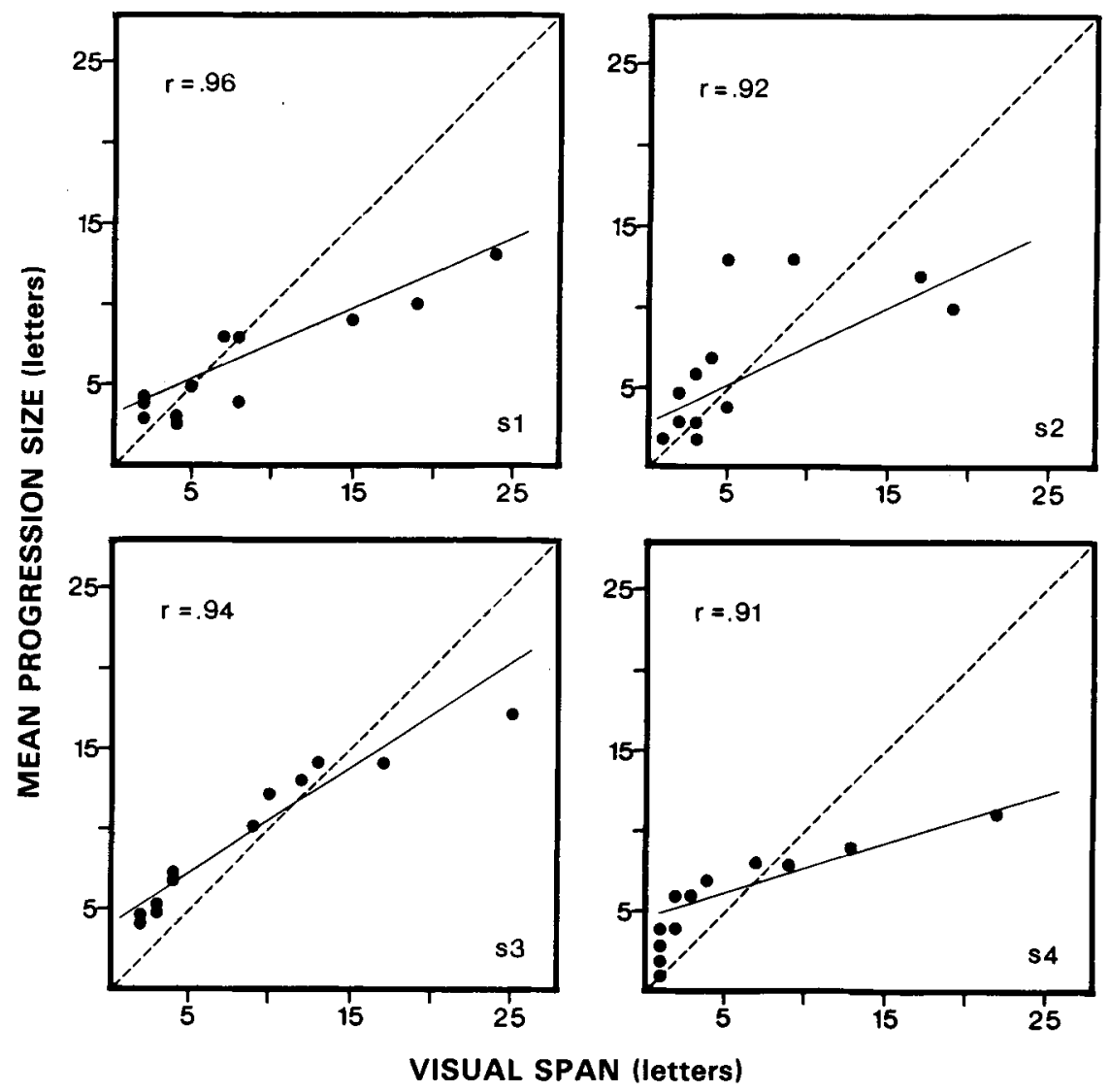

Figure 3. Visual search experiment: Correlation diagrams comparing visual span, as measured in the visibility experiment, to mean progression sizes, as measured in the search experiment, for each of the experimental visibility conditions and each of the four subjects. Each data point corresponds to the mean value observed for one experimental condition. The dashed diagonal lines represent the ideal prediction. The solid lines are actual linear regression lines. 
a
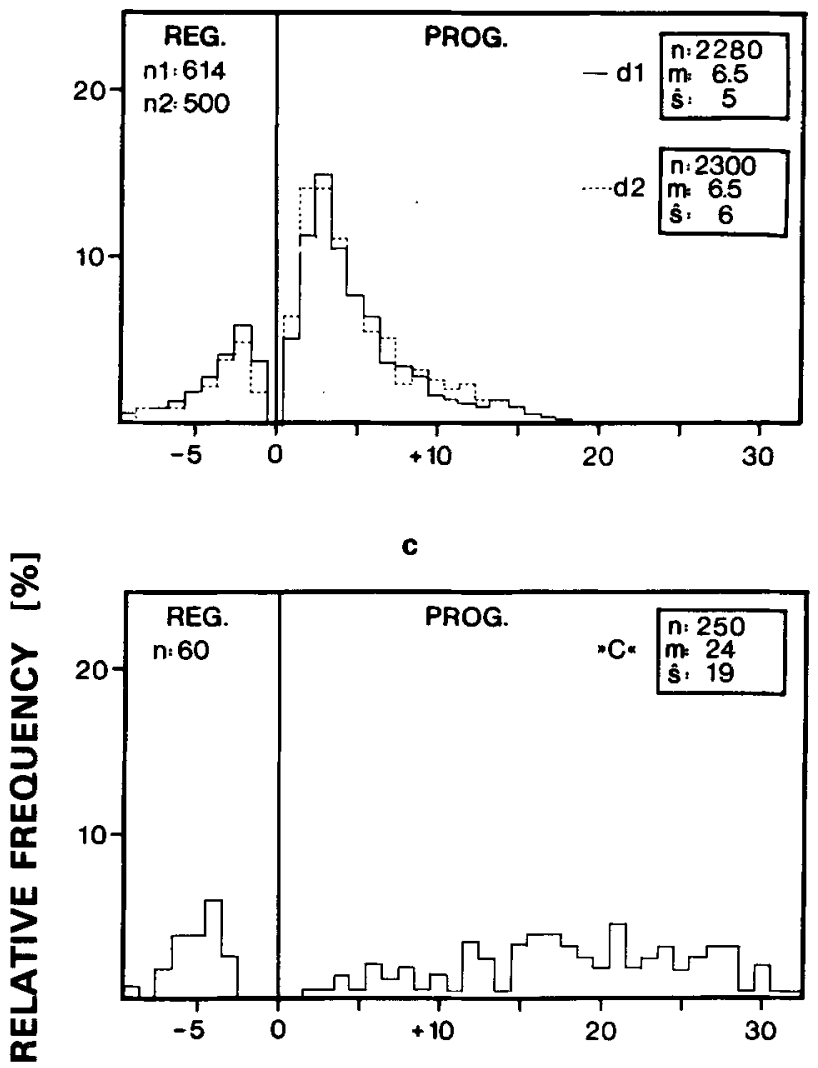

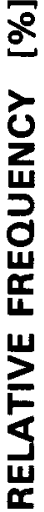


b

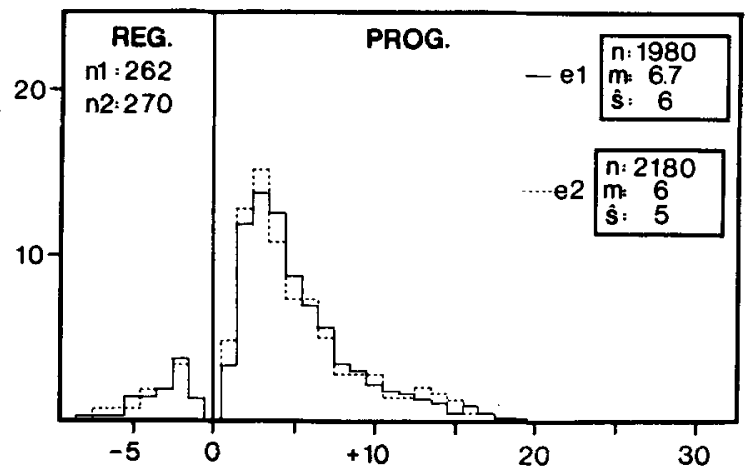

d

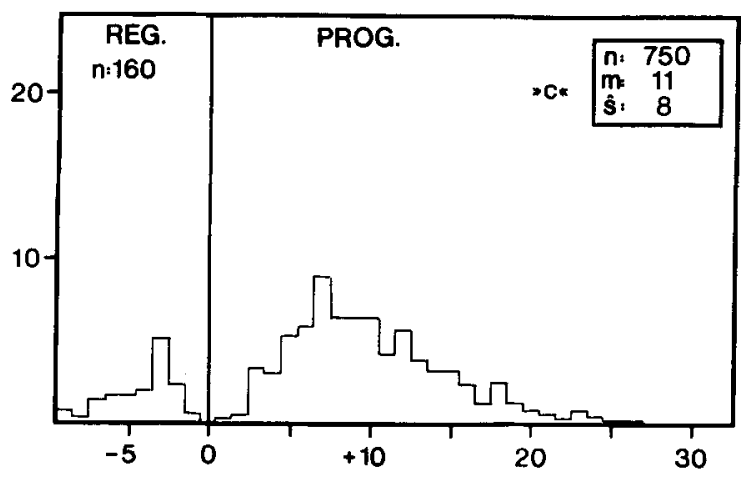

f

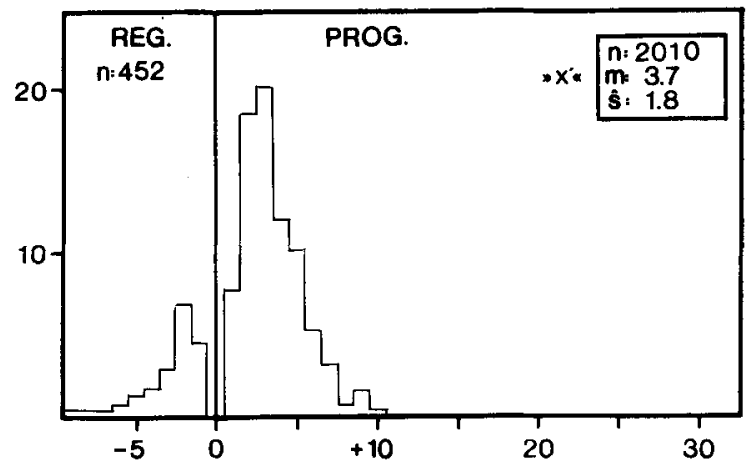

SACCADE SIZE [letters]

Figure 4. Relative frequencies for mean saccade sizes, in number of letters skipped, in the visual search experiment, as a function of viewing distance (a), interletter spacing (b), and target-background similarity (c-f). The class width is one letter. As in Figure 2, d1, d2, e1, e2, and $\mathrm{C}$ to $\mathrm{x}$ denote the different levels of distance (d), spacing (e), and similarity. $\mathrm{n}$ is the total number of data; $\mathrm{m}$, the mean; and $\mathrm{s}$, the variance. For regression amplitudes, only the total number " $\mathrm{n} 1$ and $\mathrm{n} 2$ " is indicated for comparison with progression number.

get). This variability is not due to intersubject variability: As the correlation diagrams indicate, intersubject variability was weak, and an analysis of individual histograms showed that they have the same shapes as in Figure 4. Besides, there is no particular reason why intersubject variability should vary with target-background similarity. Now, the hypothesis of direct visual span control suggests that progression sizes should center around a mean value that corresponds approximately to the visual span, for whatever target letter or visibility condition. The data of Figure $4(c-f)$ have mean values consistent with the hypothesis, but the differences in the variability of the 
histograms remains unexplained. This interesting result questions the applicability of deterministic hypotheses about oculomotor control like the one used.

Variable precision of the spatial oculomotor adjustments as a function of the strength of sensory constraints or of processing requirements. The data of the present analysis can be understood in the following way: it seems reasonable to think that the less constraining the visibility conditions are, the more "degrees of freedom" the visuomotor system has in adjusting its spatial behavior to the limits of the visual span. As a consequence, more saccades are free to vary in size: Our data seem to indicate that, in the case of targets that are easily detectable in peripheral vision ( $\mathrm{C}$ and $\mathrm{c}$ ), the visuomotor system can behave relatively liberally in the positioning of eye fixations within spatial visibility limits. The risk of missing a target letter, the uncertainty about its presence in periphery, is minimal. So there is no absolute need to adjust each saccade accurately to the visual span, that is, to scan the lines strictly and regularly "span by span." Such a precise saccade programming mode characterized by a high-aiming consistency across successive saccades consumes much processing and control energy (or time) (Kapoula, 1984), and therefore is adopted only when constraints are strong. This occurs when targets are difficult to detect in periphery because of their high similarity with the $\mathrm{xs}$ ( $\mathrm{k}$ and " $\mathrm{x}$ ") ). Here, the visuomotor system is constrained to adjust saccade sizes more precisely to the visibility limits. Otherwise, it would pay the price of missing a certain number of targets (which is discouraged by the instruction given to the subjects and by the test following each trial) or of making a large number of regressive saccades in order to verify that no target was overseen. Figure $4(\mathrm{c}-\mathrm{f})$ shows clearly that, first, there are relatively few regressions (about $10 \%$ ), and second, the ratio of number of progressions to number of regressions is constant across targets. Thus, it is tempting to consider that visual span control is strong when the task is difficult and weak when it is easy. In other words, the precision of the spatial adjustments of oculomotor behavior in such a task varies with the strength of the sensory constraints or processing requirements.

Further evidence for this idea comes from the study of the temporal characteristics of eye-movement behavior.

Analysis of the temporal adjustments of search behavior (fixation durations). Concerning fixation durations, the hypothesis of direct control by visual span allows no precise predictions. A priori, there are three possibilities concerning the way in which fixation durations could behave when visual span changes. A first hypothesis, which will be called the "quantitative control hypothesis," considers that the duration of a fixation depends on the number of letters or the amount of information processed. This would predict that the larger the visual span (or mean progression size) is, the longer the corresponding fixation duration should be. Given the homogeneous stimulus material we used, this is probably not the most plausible hypothesis, although it might hold under different conditions (O'Regan et al., 1983). A second possibility will be called the "qualitative control hypothesis," which predicts exactly the opposite relation between span and fixation durations. It considers that, in a situation like ours, fixation durations depend mainly on the ease of sensory processing. So, when visibility becomes worse and visual span becomes smaller, fixations are expected to increase (Breitmeyer, 1983; O'Regan et al., 1983). Finally, there is a third possibility, called "the tradeoff hypothesis": Provided that the visuomotor system correctly adjusts its spatial characteristics to the changing visibility conditions, for example, by making smaller saccades when visual span decreases, one might consider that there is no absolute need to simultaneously adjust the temporal characteristics. This would predict fixation durations to be largely independent of visual span changes given the results concerning the spatial adjustments shown above.

The ANOVA revealed that the two factors: viewing distance $[F(1,3)=13.3, p<.05]$ and similarity $[F(3,9)=$ $25.4, \mathrm{p}<.005]$ influenced fixation durations strongly. There was no effect of spacing $[F(1,3)=.45$, n.s. $]$. As to the similarity and spacing effects, our results again seem to be consistent with those of Prinz and Kehrer (1982) and of Rayner and Fisher (1985). The viewing distance effect replicates recent findings of Morrison and Rayner (1981) and O'Regan et al. (1983).

To test the three hypotheses considered above more directly, fixation durations were plotted in Figure 5 against visual span, following the same principle as that used in Figure 3.

In general, the correlations are weaker between fixation duration and visual span than they are for mean progression amplitude and visual span. Nevertheless, there seems to be a certain negative relationship between the two variables. Three out of four correlations are significant (s1, s2, s4). However, on average, the coefficient of determination, $\mathrm{r}^{2}$, is .45 , so we can say that only about $45 \%$ of the variance in fixation durations are accounted for by changes in visual span. Correlations were also computed on the basis of nonlinear regressions. The results were much the same as for linear regressions, and mean $\mathrm{r}^{2}$ did not change significantly. Moreover, the data seem to indicate two distinct classes of durations with differing variability within each class rather than a continuous linear or nonlinear function. This might reflect two oculomotor strategies with different timing characteristics, as discussed below.

Sensory and/or cognitive effects upon fixation durations? One thing becomes immediately clear in this last analysis: Fixation durations certainly do not increase with visual span, and the "quantitative control hypothesis" must be rejected in this case. So one can consider that in a task like this, which uses a homogeneous background, it is not the amount of information (in terms of number of letters) processed during a fixation which acts upon its duration, although, with a heterogeneous background, things might be different (Lévy-Schoen et al., 1984). On 


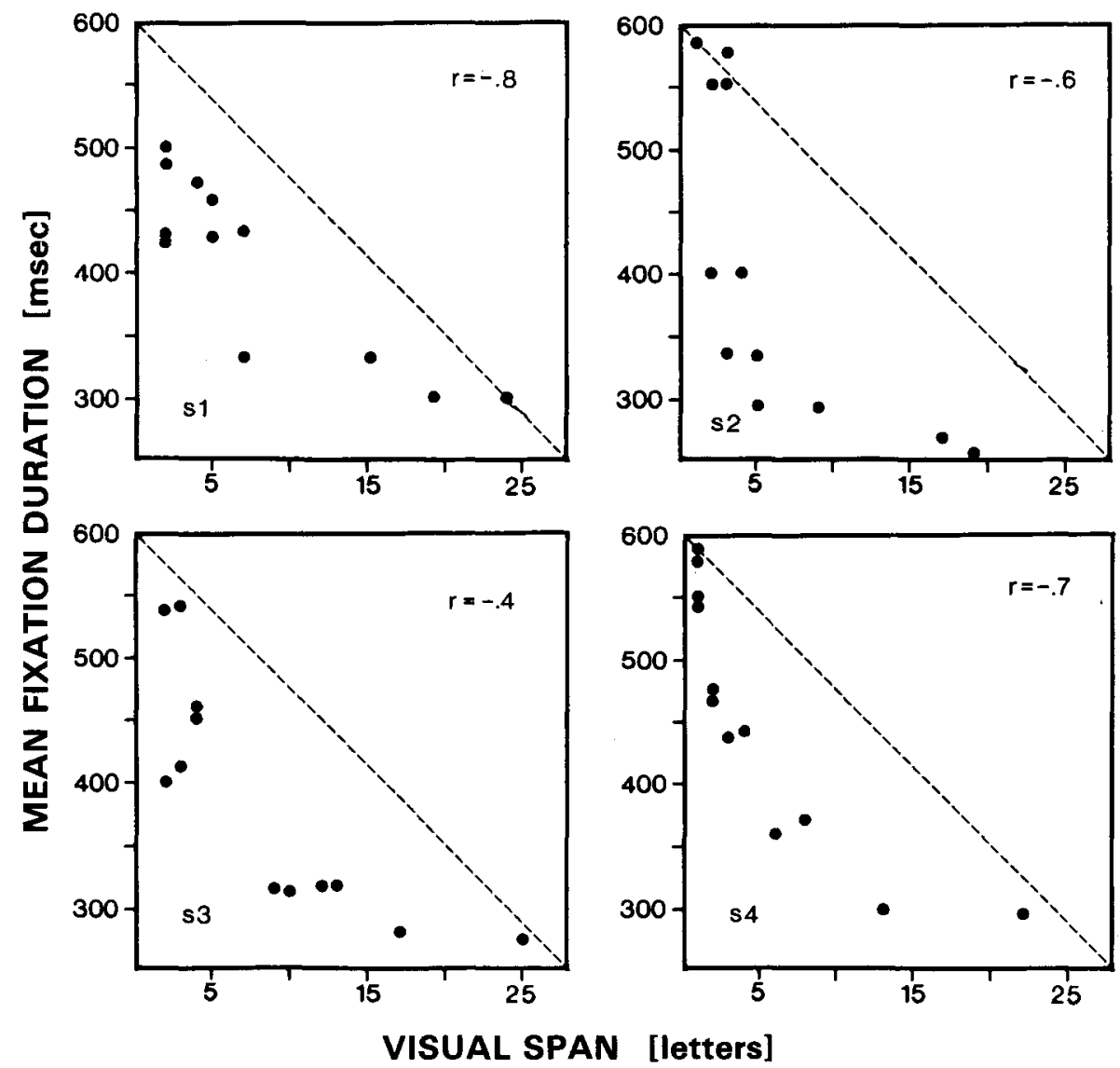

Figure 5. Visual search experiment: Correlation diagrams comparing visual span, as measured in the visibility experiment, to mean fixation duration, as measured in the search experiment for each of the experimental conditions and for each subject. Each data point corresponds to the mean value observed for one experimental condition.

the other hand, one cannot really decide whether the "qualitative control hypothesis" or the "tradeoff hypothesis" is correct, since there is no clear negative relationship between span and fixation durations $(45 \%$ of variance determined by changes in visual span leave $55 \%$ determined by other factors whose nature is not yet clear). Rather, the fixation duration data analyzed in Figure 5 seem to be separable into two different classes: short and constant fixation durations if span is superior to about five letters, and longer, variable durations as span decreases to less than five letters. This might indicate that two distinct oculomotor strategies with different timing characteristics are used. This is supported by Figure $6(a-c)$, which gives relative frequency histograms for fixation durations as a function of viewing distance (a), interletter spacing (b), and target-background similarity (c). Three points are especially interesting in these results:

First, fixation durations increase about 20 to $40 \mathrm{msec}$, when viewing distance doubles from 60 to $120 \mathrm{~cm}$, independently of what spacing or target letter is used (there was no significant interaction between the three factors). This distance effect upon fixation durations is consistent with the findings of other recent studies, in which differ- ent materials, tasks, and subjects were used and the effect had a similar amplitude-about $20 \mathrm{msec}$ in Morrison and Rayner (1981) and about $30 \mathrm{msec}$ in O'Regan et al. (1983). The amplitude of this viewing distance effect, as well as the fact that it is apparently independent of stimulus material and task, might suggest that it is sensory in nature. There are good reasons to think so: doubling viewing distance reduces the retinal stimulus image to half its size or increases the spatial frequencies in the stimulus spectrum. Higher spatial frequencies need additional processing time, probably at the level of signal transmission times (Breitmeyer, 1975, 1983; Vassilev \& Mitov, 1976).

But there seems to be a mystery about the effect of viewing distance on eye movements: Since Javal's (1879) early observations, a number of studies failed to demonstrate a viewing distance effect upon saccade sizes (measured in number of letters, of course) in reading (Morrison \& Rayner, 1981; O’Regan et al., 1983; Paterson \& Tinker, 1947). Yet, viewing distance slightly but systematically influences visual span, as measured in letter identification or detection tasks. On the other hand, it has been shown that fixation durations are found, in all of the 
a



ํํㄴ

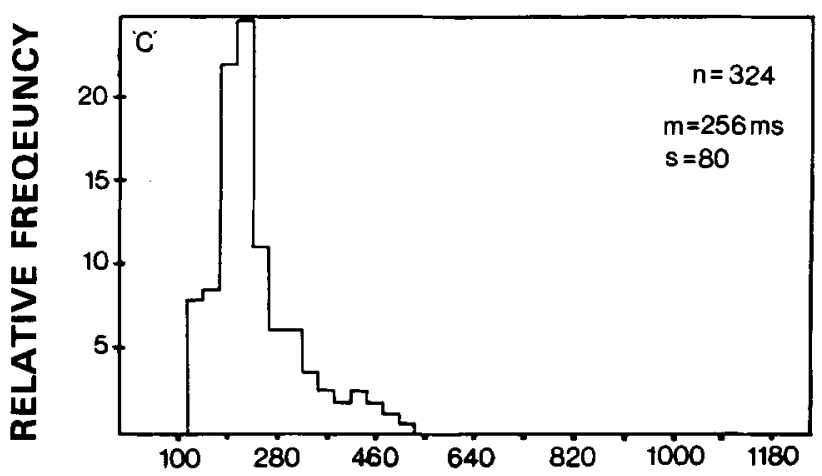

e

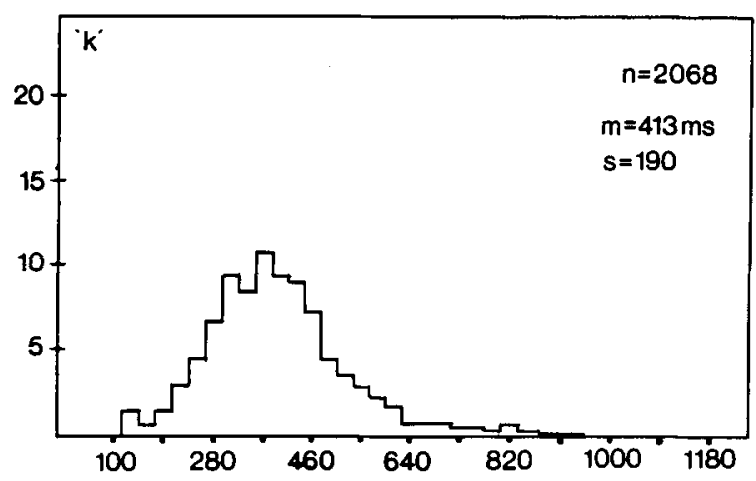

b

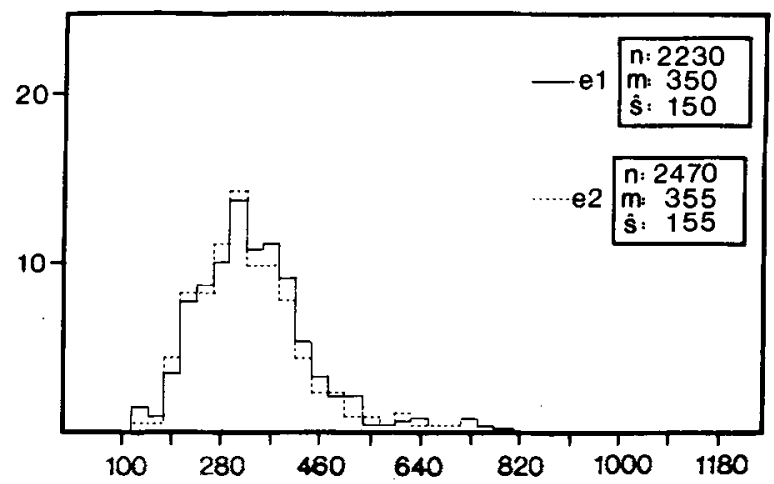

d

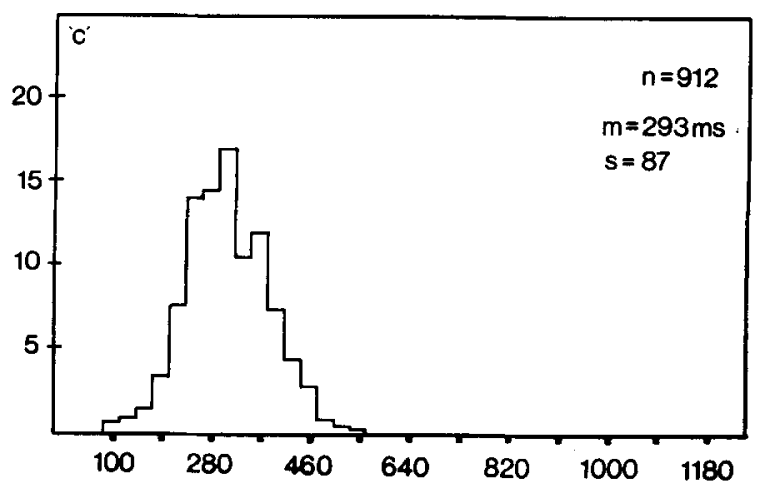

f

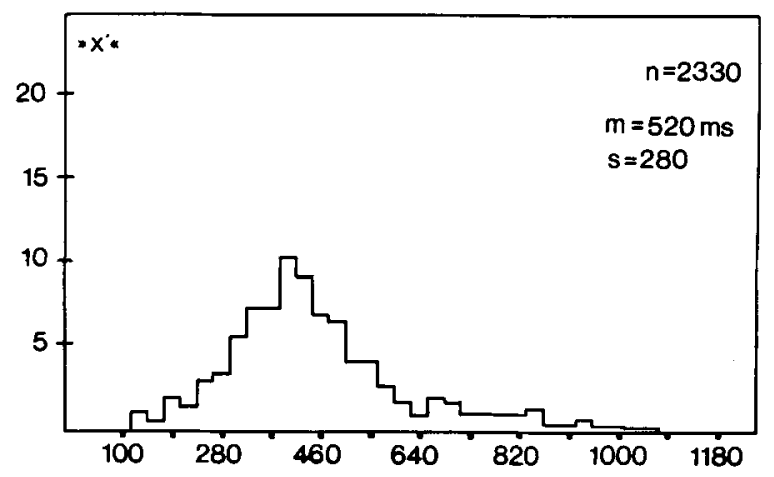

FIXATION DURATION [msec]

Figure 6. Visual search experiment: Relative frequencies for mean fixation durations, in milliseconds, as a function of viewing distance (a), interletter spacing (b), and target-background similarity (c-f). The class width is $\mathbf{3 0}$ msec. The different conditions are denoted as in Figures 2 and 4: $n$ is the total number of data, $m$ the mean, and $s$ the variance.

studies mentioned above, to increase systematically with viewing distance, a fact which has been interpreted in terms of a sensory effect. Since stimulus exposure duration is always kept constant, in the visibility experiments on static visual span, across viewing distance, there is the following interpretation of why an effect is found on visual span but not on saccade sizes: Within an intermediate range, viewing distance acts mainly upon the temporal processing characteristics (see studies mentioned above). If, in the psychophysical letter-detection tasks used to determine visual span, stimulus exposure durations are kept constant and viewing distance is varied, this results in a decline of visual span; contrary to the dynamic visual search situation. it is not possible for the visual system, 
in these tasks, to compensate for the worse visibility condition by increasing processing times. However, if stimulus exposure durations in this kind of letter-detection task were matched with the varying viewing distances, for example, by using longer exposure durations for the longer viewing distances, then, following the above observations and theoretical arguments (Breitmeyer, 1975, 1983; Vassilev \& Mitov, 1976), we could expect visual span to remain constant, within a certain range, across viewing distances. Recent investigations performed by our research group in collaboration with A. Barszcz suggest that visual span depends on exposure duration, but we have not yet studied the interaction with viewing distance. Clearly, further research on this issue is needed.

The second result contained in Figure 6 is that fixation durations do not change when interletter spacing is doubled from 4 to 8 pixels. One might expect that they should decrease, at least slightly, because of the reduced contour interaction and lateral masking in the large-spacing condition. But data reviewed by Moffitt (1980) suggest that for the comparatively small spacing values we used, there are generally no significant effects on fixation durations in continuous visual search tasks. Furthermore, the findings of Prinz and Kehrer (1982) and Rayner and Fisher (1985) also suggest that item density does not strongly affect search time when the characteristics of the target or distractor items are manipulated simultaneously.

The third important result here, which is consistent with the idea of two oculomotor strategies with different timing characteristics indicated by the correlation diagram data, is that fixation durations almost double and become more dispersed as the target letter changes from $C$ to " $\mathrm{x}$ " " and when spans mean progression size decreases dramatically. This might be explained in terms of a purely sensory effect due to the worse visibility conditions. However, in fact, in both conditions (e.g., $\mathrm{C}$ and " $\mathrm{x}$ "'), the subjects scanned physically identical lines, namely, lines containing only xs (only data coming from lines without any targets were selected for analysis, as pointed out before). The only thing that changed from one condition to the other was the expectation and the level of uncertainty about the target's presence in the line, not sensory information. The observed changes in fixation duration as a function of the similarity factor cannot thus have been due to a modification of sensory mechanisms. Rather, our claim is that higher level, cognitive processes were involved in the decision about the target's presence within the zone of visibility and in the computation of the spatial parameters of saccades, and that these were modulated when target-background similarity changed (Jacobs, 1985b; see also Vaughan \& Graefe, 1977). The progression amplitude results shown in histograms $c-f$ of Figure 4 were interpreted by considering that the precision of the spatial adjustments to visual span (the aiming consistency across successive saccades) increases as processing requirements become stronger (targets become more difficult to detect in peripheral vision).

Now, the present fixation duration differences can be understood in the following way: on the one hand, recent models of eye-movement control by two largely independent subsystems functioning in parallel (Becker \& Jurgens, 1979; Deubel, 1984; Findlay, 1983; Jacobs, 1985b; Morrison, 1984; Rayner, Slowiaczek, Clifton, \& Bertera, 1983; see also Rayner \& McConkie, 1976) suggest that the saccade amplitude that is being elaborated by the "where" system depends on the moment the "when" system triggers the saccade, independently of whether the amplitude computation is already terminated or not. In the latter case, an imperfect neuronal impulse would be sent to the eye muscles, resulting in an inaccurate saccade. In the case of easy targets ( $\mathrm{C}$ and $\mathrm{c}$ ), the visuomotor system seems to choose a strategy that adjusts saccade sizes liberally to visual span and thus minimizes the times for processing the sensory information and for controlling the spatial parameters of saccades. Given the minimal risk of missing a target, the lines are scanned rapidly, with a minimum of cognitive effort (compare discussion of saccade size histograms above). This results in relatively short and constant fixation durations, as supported by Figures $6 \mathrm{c}$ and $6 \mathrm{~d}$, but in very variable saccade sizes (see Figures $4 \mathrm{c}$ and $4 d$ ). Following the abovementioned saccade-control models, such a liberal "minimization strategy" might imply that a portion of saccades is triggered even before the amplitude computation in the "where" system is accomplished, resulting in inaccurately adjusted saccades (Jacobs, 1985b; cf. Morrison, 1984). Thus, the large variability of saccade amplitudes compared with the small variance in fixation durations for these "easy" conditions could be accounted for (see Figures 4c, 4d, 6c, and 6d).

On the other hand, following recent findings on the control of target-directed saccades (Coeffe, 1985; Findlay, 1983; Kapoula, 1984), it seems reasonable to think that adjusting saccade sizes precisely to visual span might require more cognitive effort, processing energy, and computational time than the relatively liberal adjustment under the minimization strategy in the case of the easily detectable targets. Our data for the difficult targets $(\mathrm{k}$ and " $x$ "'), for which saccade amplitudes are relatively small and constant (see Figures $4 \mathrm{e}$ and $4 \mathrm{f}$ ) but fixation durations long and variable (Figures 6e and 6f), support this idea. Indeed, here a strategy that minimizes cognitive effort and processing times would not be efficient: If saccades were badly adjusted to the visual span, either some targets would be missed or more regressive movements would become necessary (verification saccades). As discussed above, this is not the case. Instead, the visuomotor system apparently needs much time at each fixation to determine the target's presence/absence within the zone of visibility and to elaborate the motor command, resulting in regular, well-adjusted saccade sizes. It seems plausible that under these conditions oculomotor decision times vary a lot more than under a "minimization strategy."

This interpretation, which reconciles the experimental data for mean progression sizes and fixation durations from the correlation diagrams and the histograms, might appear somewhat speculative. But it turns our attention to the more complex cognitive control processes that seem 
to be involved in a simple search task like the one used, in which, in the first place, visuosensory factors were called into play.

\section{CONCLUSION}

The present experiments continued a series of studies that had focused on exploring the role of elementary visuosensory constraints that determine the number of letters visible at each fixation for eye-movement control processes in reading and visual search (Jacobs, 1985a; Lévy-Schoen et al., 1984; O'Regan et al., 1983). In this study, a particular, very simplified stimulus material and task were used in order to discard, as much as possible, the influences of cognitive factors, such as information load, on eye-movement control. The somewhat extreme hypothesis was made that, under such simplified conditions, saccade sizes should directly parallel changes in visual span, as measured in a psychophysical experiment under various visibility conditions. Under this hypothesis, at each fixation, the eyes should be sent to an area on the line where new visual information is available, that is, the limit of visual span. The major finding of this study is that, in such a task, saccades are determined by visual span, but that control is less direct than the extreme working hypothesis suggested. The postulated deterministic eye-movement control rule- " at each moment jump to the limit of visual span"-is not strictly respected by the visuomotor system in all the different visibility conditions. Adjustment of saccade sizes to visual span improves as the sensory constraints become stronger. If the constraints are relatively weak (and span is large: e.g., targets $\mathrm{C}$ and c), we find very variable saccade sizes but very constant and short fixation durations. This indicates probably that, under such conditions, the visuomotor system minimizes the times for processing the sensory information and for preparing the spatial parameters of the saccades. If processing requirements become stronger (targets $\mathrm{k}$ and " $x$ "'), the opposite result is shown: saccade sizes are small and very constant, but fixation durations become very long (up to $600 \mathrm{msec}$ ) and more dispersed. Probably, under these constraints, much time is needed to make a decision about the target's presence/absence within the zone of visibility and for programming precisely adjusted saccades.

As yet, we do not know much about the sensorimotor and cognitive mechanisms that function on the basis of these oculomotor adjustments (Jacobs, 1985b). Further research using even more simplified tasks than the one used here (Kowler, 1985; Rayner et al., 1983) will be needed to advance our comprehension of elementary eyemovement control processes.

\section{REFERENCES}

BECKER, W., \& JURGENS, R. (1979). An analysis of the saccadic system by means of double step stimuli. Vision Research, 19, 967-983. Bouma, H. (1978). Visual search and reading: Eye movements and func- tional visual field: A tutorial review. In J. Requin (Ed.), Attention and performance, VII (pp. 115-146). Hillsdale, NJ: Erlbaum.

BREITMEYER, B. G. (1975). Simple reaction time as a measure of the temporal response properties of transient and sustained channels [Letter to the editor]. Vision Research, 15, 1411-1412.

BreitMeyer, B. G. (1983). Sensory masking, persistence, and enhancement in visual exploration and reading. In $\mathrm{K}$. Rayner (Ed.), Eye movements in reading: Perceptual and language processes (pp. 3-30). New York: Academic Press.

COEFFE, C. (1985). Two ways of improving accuracy of the saccade. Paper presented at the 3rd European Conference on Eye Movements, Paris-Dourdan.

Deubél, H. (1984). Wechselwirkung von Sensorik und Motorik bei sakkadischen Augenbewegungen. Eine kybernetische Beschreibung des visuellen Systems. Unpublished doctoral thesis, University of Munich.

ENGEL, F. L. (1977). Visual conspicuity: Visual search and fixation tendencies of the eye. Vision Research, 17, 95-108.

FindLAY, J. M. (1983). Visual information processing for saccadic eye movements. In A. Hein \& M. Jeannerod (Eds.), Spatially oriented behavior. New York: Springer.

Finney, D. J. (1947). Probit analysis. Cambridge: University Press. Humbe RT, R. (1985). Comparaison par simulation de méthodes adaptatives de mesure de seuil. Bulletin de la Société Francaise d'Optique Physiologique, 4, 2

JACOBS, A. M. (1985a). Facteurs sensoriels et cognitifs dans l'exploration visuelle. In J. P. Caverni, C. Bonnet, \& J. P. Codol (Eds.), Hommage à Georges Noizet: Jugement et langage. Bulletin de Psychologie, No. spécial.

JACOBS, A. M. (1985b). Toward a model of oculomotor control in visual search. Paper presented at the 3rd European Conference on Eye Movements, Paris-Dourdan.

JaVAL, E. (1879). Essai sur la physiologie de la lecture. Annales d'Oculistique, 82, 243-253.

KAPOULA, Z. (1984). Aiming precision and characteristics of saccades. In A. G. Gale \& F. Johnson (Eds.), Theoretical and applied aspects of eye movement research. Amsterdam: North Holland.

KEREN, G., \& BAGGEN, S. (1981). Recognition models of alphanumeric characters. Perception \& Psychophysics, 29, 234-246.

Kowler, E. (1985). Programming sequences of saccades. Paper presented at the 3rd ECEM, Paris-Dourdan.

Lévy-Schoen, A., O'Regan, J. K., Jacobs, A. M., \& Coeffe, C. (1984). The relation between visibility span and eye movements in various scanning tasks. In A. G. Gale \& F. Johnson (Eds.), Theoretical and applied aspects of eye movement research (pp. 133-142). Amsterdam: North Holland.

MACKWORTH, N. H. (1976). Stimulus density limits the useful field of view. In R. A. Monty \& J. W. Senders (Eds.), Eye movements and psychological processes. Hillsdale, NJ: Erlbaum.

MCConkIE, G. W. (1983). Eye movements and perception during reading. In $\mathrm{K}$. Rayner (Ed.), Eye movements in reading: Perceptual and language processes (pp. 65-93). New York: Academic Press.

McConkiE, G. W., \& RAYNER, K. (1975). The span of the effective stimulus during a fixation in reading. Perception \& Psychophysics, 17, 578-586.

MoffitT, K. (1980). Evaluation of the fixation duration in visual search. Perception \& Psychophysics, 27, 360-372.

Morrison, R. E. (1984). Manipulations of stimulus onset delay in reading: Evidence for parallel programming of saccades. Journal of Experimental Psychology: Human Perception \& Performance, 10 , 667-682.

Morrison, R. E., \& Rayner, K. (1981). Saccade size in reading depends upon character spaces and not visual angle. Perception \& Psychophysics, 30, 395-396.

O'REGAN, J. K. (1975). Structural and contextual constraints on eye movements in reading. Unpublished doctoral thesis, University of Cambridge.

O'Regan, J. K. (1979). Saccade size control in reading: Evidence for the linguistic control hypothesis. Perception \& Psychophysics, 25, 501-509.

O'Regan, J. K. (1983). Elementary perceptual and eye movement control 
processes in reading. In. K. Rayner (Ed.), Eye movements in reading: Perceptual and language processes (pp. 121-138). New York: Academic Press.

O'Regan, J. K., Lévy-Schoen, A., \& Jacobs, A. M. (1983). The effect of visibility on eye movement parameters in reading. Perception \& Psychophysics, 34, 457-464.

Paterson, D. G., \& Tinker, M. A. (1947). The effect of typography upon perceptual span in reading. American Journal of Psychology, 3, 388-396.

Prinz, W., \& KeHRER, L. (1982). Recording detection distances in continuous visual search. In R. Groner \& P. Fraisse (Eds.), Cognition and eye movements (pp. 10-23). Berlin: Verlag der Deutschen Wissenschaften.

RAYNER, K. (1983). The perceptual span and eye movement control during reading. In $\mathrm{K}$. Rayner (Ed.), Eye movements in reading: Perceptual and language processes (pp. 97-118). New York: Academic Press.

RAYNER, K., \& FISHER, D. F. (1985). Eye movements and the perceptual span during visual search. Paper presented at the 3rd European Conference on Eye Movements, Paris-Dourdan.
RAYNER, K., \& McConkIE, G. W. (1976). What guides a reader's eye movements. Vision Research, 16, 829-837.

Rayner, K., Slowiaczek, M. L., Clifton, C., \& Bertera, J. H. (1983). Latency of sequential eye movements: Implications for reading. Journal of Experimental Psychology: Human Perception \& Performance, 9, 912-922.

Taylor, M. M., \& Creelman, C. D. (1967). PEST: Efficient estimates on probability function. Journal of the Acoustical Society of America, 41, 782-787.

VAssilev, A., \& Mitov, D. (1976). Perception time and spatial frequency. Vision Research, 16, 89-92.

VAughaN, J., \& Graefe, T. M. (1977). Delay of the stimulus presentation after the saccade in visual search. Perception \& Psychophysics, 22, 201-205.

WATt, R. J., \& ANDREWS, D. P. (1981). APE: Adaptive probit estimation of psychometric function. Current Psychological Reviews, 1 , 205-214.

(Manuscript received August 8, 1985; revision accepted for publication December 3,1985 .) 